\title{
Population structure and morphometric variance of the Apis mellifera scutellata group of honeybees in Africa
}

\author{
Sarah Radloff' and Randall Hepburn ${ }^{2}$
}

\begin{abstract}
The honeybee populations of Africa classified as Apis mellifera scutellata Lepeletier were analysed morphometrically using multivariate statistical techniques. The collection consisted of nearly 15,000 worker honeybees from 825 individual colonies at 193 localities in east Africa, extending from South Africa to Ethiopia. Factor analysis established one primary cluster, designated as A. $m$. scutellata. Morphocluster formation and inclusivity (correct classification) are highly sensitive to sampling distance intervals. Within the $A$. $m$. scutellata region are larger bees associated with high altitudes of mountain systems which are traditionally classified as $A$. $m$. monticola Smith, but it is evident that these bees do not form a uniform group. Variance characteristics of the morphometric measurements show domains of significantly different local populations. These high variance populations mostly occur at transitional edges of major climatic and vegetational zones, and sometimes with more localised discontinuities in temperature. It is also now evident that those $A$. $m$. scutellata introduced nearly fifty years ago into the Neotropics were a particularly homogenous sample, which exhibited all the traits expected in a founder effect or bottleneck population.
\end{abstract}

\section{INTRODUCTION}

Modern studies of the classification of the honeybees of Africa stem from the work of Kerr and PortugalAraújo (1958). They recognised five subspecies, the most widely spread of which was $A$. $m$. scutellata (then called $A$. m. adansonii) and its various ecotypes (Kerr, 1992). Details of the nomenclatural history are reviewed by Ruttner (1988) and Daly (1991). Further analyses of the bees falling under the A. $m$. scutellata umbrella of distribution were provided by Smith (1961) and in a series of studies summarised by Ruttner (1988). Recently the large morphometric databases on the honeybees of Africa that had been separately assembled in Germany and South Africa were amalgamated into a single unit of considerable depth and breadth and the apifauna of the whole continent morphometrically re-analysed (Hepburn and Radloff, 1998).

In general terms the results of Hepburn and Radloff (1998) supported many of the interpretations of Ruttner (1988), but some not unexpected refinements were made. One was the fact that all of the bees classified as $A . m$. scutellata on the basis of multivariate morphometric analyses by Ruttner (1988) resolved into two distinct morphoclusters in the analysis of Hepburn and Radloff (1998). The honeybees of southern Ethiopia and northern Kenya formed one A. m. scutellata-like morphocluster that differed from the morphocluster to the south, in Zimbabwe and South Africa (whence the Neotropical introductions originated). These morphoclusters were simply designated as "scutellata 1 " and "scutellata 2 " pending the acquisition of additional data.
This A. m. scutellata problem is interesting for several different reasons. Firstly, the geographic distribution of the Ruttner A. m. scutellata was more or less rectangular, extending the length of the eastern highlands of Africa from South Africa to Ethiopia (hence the name "highland bee"). In the Hepburn-Radloff analysis this rectangle was somewhat crimped or narrow-waisted where $A$. $m$. adansonii pushed eastwards from Zambia and $A$. $m$. litorea westwards from Mozambique. Secondly, in both the Ruttner (1988) and Hepburn and Radloff (1998) studies there was an extreme paucity of material then available from northwestern Mozambique, western Zimbabwe, eastern Botswana and southern Kenya. Tanzania, Malawi and Lesotho were simply dataless gaps. Thirdly, until now there was not sufficient information on the original mother populations of the bees subsequently introduced into the Neotropics, from which reasonable inference might have been made.

It has recently been established for the African apifauna that morphocluster formation resulting from multivariate analyses can be very sensitive to sample distance intervals as well as to levels of statistical confidence employed to make interpretations (Radloff and Hepburn, 1998). Very recently a considerable amount of new material has been obtained from Botswana, Zimbabwe, Malawi, Lesotho and Tanzania. Here we report the results of fresh analyses of a considerably (30\%) enhanced database to re-examine the honeybee populations of the A. m. scutellata group, their various characteristics, biogeographical relationships and areas of hybridisation with neighbouring populations of other sub- 
species. The geographical area investigated was the whole of Ruttner's original A. m. scutellata rectangle, extending from South Africa to Ethiopia (Figure 1). Also, to the extent possible, Palaeotropical A. m. scutellata were compared with recent Neotropical (but non-morphometric) data to estimate the extent of bottlenecking (expression of a founder effect of the original introductions) in the latter region.

\section{MATERIAL AND METHODS}

Recently (1997) the morphometric databases on honeybees of the Institut für Bienenkunde (Ruttner collection, Oberursel, Germany) and of the Apiculture Group at Rhodes University (Hepburn and Radloff collection, Grahamstown, South Africa) were amalgamated to form a single database for Africa. A new multivariate morphometric analysis of

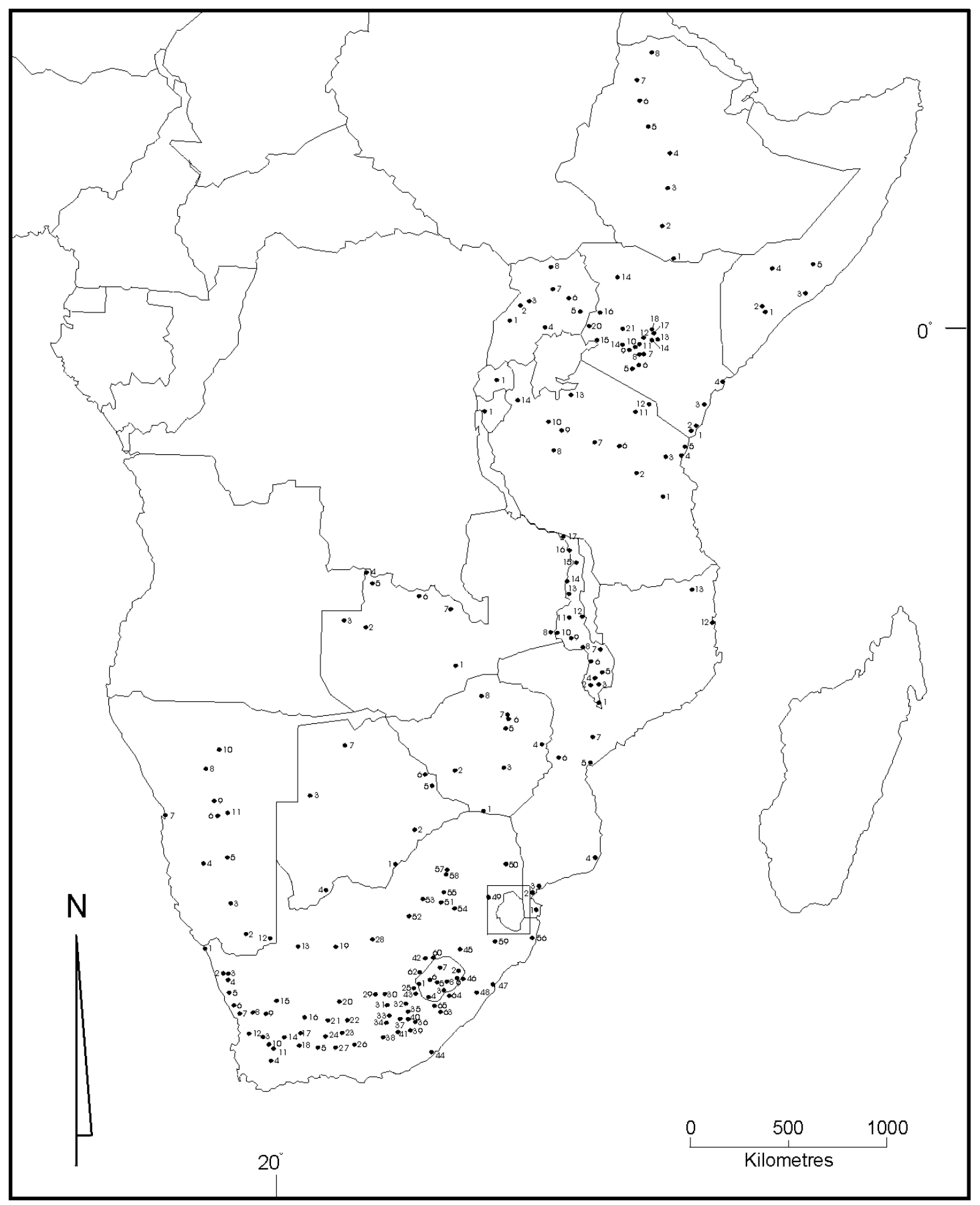

Figure 1 - Map of eastern Africa indicating the localities at which worker honeybees were collected for analysis in our study. Note that for each country the numbering system for localities always begins with " 1 " to avoid overlapping. Map numbers for each country correspond to the geographical and biological information in Table I. 
this material was recently published (Hepburn and Radloff, 1998). In the interim, important new collections of honeybee workers were obtained from Botswana, Lesotho, Malawi, Tanzania and Zimbabwe, that increased the original database by $30 \%$. This material has been measured, inserted into the database and a fresh multivariate morphometric analysis of the honeybees of the eastern side of Africa made.

All of the worker bees used in the study were sampled from the colonies of small-scale, fixed-site beekeepers at 193 localities, extending the length of eastern Africa from South Africa to Ethiopia (Table I). While "captive" colo- nies were often sampled it must be understood that the bees are simply attracted to empty hives from the wild population. Bees in Africa are very rarely transported and bee breeding is virtually non-existent. Thus, the samples used in the analysis constitute authentic subsamples of the wild population whence they came. Morphometric measurements were usually taken on 20 worker bees per colony from a variable number of colonies per locality, total colonies sampled being 825 individual colonies. A total of 14,973 individual worker honeybees was measured morphometrically (Table I).

Table I - Distribution of the localities, co-ordinates, altitudes, sample sizes and intercolonial variances of worker honeybees analysed morphometrically.

\begin{tabular}{|c|c|c|c|c|c|c|c|}
\hline \multirow[b]{2}{*}{$\begin{array}{l}\text { Countries } \\
\text { and localities }\end{array}$} & \multirow{2}{*}{\multicolumn{2}{|c|}{ Co-ordinates }} & \multirow[b]{2}{*}{ Altitude (m) } & \multicolumn{2}{|c|}{ Sample sizes } & \multirow[b]{2}{*}{ Variance } & \multirow[b]{2}{*}{$\begin{array}{l}\text { Map } \\
\text { Ref. }\end{array}$} \\
\hline & & & & Colonies & Bees & & \\
\hline \multicolumn{8}{|l|}{ Botswana } \\
\hline Francistown & $21.11 \mathrm{~S}$ & $27.32 \mathrm{E}$ & 900 & 4 & 80 & 13.7 & 5 \\
\hline Gaborone & $24.45 \mathrm{~S}$ & $25.55 \mathrm{E}$ & 1000 & 5 & 100 & 6.2 & 1 \\
\hline Ghanzi & $21.39 \mathrm{~S}$ & $21.39 \mathrm{E}$ & 1137 & 1 & 20 & - & 3 \\
\hline Irdbridge & $19.15 S$ & $23.30 \mathrm{E}$ & 945 & 1 & 8 & - & 7 \\
\hline Mahalapye & $23.05 \mathrm{~S}$ & $26.51 \mathrm{E}$ & 1000 & 5 & 100 & 16.5 & 2 \\
\hline Tsabong & $26.28 \mathrm{~S}$ & $21.35 \mathrm{E}$ & 1034 & 1 & 20 & - & 4 \\
\hline Tutume & $20.26 \mathrm{~S}$ & $27.02 \mathrm{E}$ & 1100 & 4 & 80 & 23.2 & 6 \\
\hline \multicolumn{8}{|l|}{ Burundi } \\
\hline Bujumbura & $03.22 \mathrm{~S}$ & $29.19 \mathrm{E}$ & 800 & 6 & 120 & $74.3^{*}$ & 1 \\
\hline \multicolumn{8}{|l|}{ Ethiopia } \\
\hline Addis Ababa & $09.03 \mathrm{~N}$ & $38.42 \mathrm{E}$ & 2842 & 9 & 180 & 22.6 & 4 \\
\hline Adi Arkay & $13.35 \mathrm{~N}$ & $37.57 \mathrm{E}$ & 950 & 5 & 100 & 36.8 & 8 \\
\hline Agere Maryam & $05.13 \mathrm{~N}$ & $38.20 \mathrm{E}$ & 2000 & 5 & 100 & 18.5 & 2 \\
\hline Bahir Dar & $11.33 \mathrm{~N}$ & $37.25 \mathrm{E}$ & 2400 & 5 & 100 & 12.7 & 6 \\
\hline Debre Markos & $10.19 \mathrm{~N}$ & $37.41 \mathrm{E}$ & 2000 & 5 & 99 & 13.3 & 5 \\
\hline Gonder & $12.39 \mathrm{~N}$ & $37.29 \mathrm{E}$ & 2121 & 6 & 120 & 22.1 & 7 \\
\hline Mega & $04.02 \mathrm{~N}$ & $31.19 \mathrm{E}$ & 2100 & 5 & 98 & 18.9 & 1 \\
\hline Shashemene & $07.13 \mathrm{~N}$ & $38.33 \mathrm{E}$ & 1800 & 6 & 119 & 18.1 & 3 \\
\hline \multicolumn{8}{|l|}{ Kenya } \\
\hline Aberdare & $00.35 \mathrm{~S}$ & $36.38 \mathrm{E}$ & 2666 & 1 & 20 & - & 9 \\
\hline Chepkitale & $00.58 \mathrm{~N}$ & $34.33 \mathrm{E}$ & 2986 & 1 & 10 & - & 16 \\
\hline Chiokariga & $00.17 \mathrm{~S}$ & $37.55 \mathrm{E}$ & 762 & 3 & 45 & $41.4^{*}$ & 8 \\
\hline Chuka & $00.20 \mathrm{~S}$ & $37.38 \mathrm{E}$ & 1401 & 1 & 15 & - & 7 \\
\hline Gatimbi & $00.01 \mathrm{~N}$ & $37.39 \mathrm{E}$ & 1584 & 2 & 30 & 37.0 & 17 \\
\hline Kaaga & $00.04 \mathrm{~N}$ & $37.39 \mathrm{E}$ & 1600 & 3 & 45 & 3.2 & 18 \\
\hline Kerio Valley & $02.24 \mathrm{~N}$ & $36.21 \mathrm{E}$ & 450 & 1 & 20 & - & 19 \\
\hline Kimbo/Meru & $00.06 \mathrm{~N}$ & $37.29 \mathrm{E}$ & 2437 & 12 & 177 & 28.9 & 10 \\
\hline Kimititi & $00.34 \mathrm{~N}$ & $34.34 \mathrm{E}$ & 1534 & 6 & 90 & 28.0 & 20 \\
\hline Kiria & $00.12 \mathrm{~S}$ & $37.39 \mathrm{E}$ & 1371 & 1 & 15 & - & 13 \\
\hline Lake Baringo & $00.38 \mathrm{~N}$ & $36.03 \mathrm{E}$ & 980 & 1 & 12 & - & 21 \\
\hline Lamu & $02.15 \mathrm{~S}$ & $40.50 \mathrm{E}$ & 0 & 1 & 20 & - & 4 \\
\hline Malindi & $03.14 \mathrm{~S}$ & $40.05 \mathrm{E}$ & 0 & 1 & 20 & - & 3 \\
\hline Meru (township) & $00.04 \mathrm{~N}$ & $37.39 \mathrm{E}$ & 1554 & 4 & 65 & $42.2^{*}$ & 11 \\
\hline Mombasa & $04.04 \mathrm{~S}$ & $39.40 \mathrm{E}$ & 0 & 1 & 20 & - & 2 \\
\hline Mt. Elgon & $01.07 \mathrm{~N}$ & $34.31 \mathrm{E}$ & 4320 & 8 & 125 & 28.2 & 15 \\
\hline Mt. Kenya & $00.32 \mathrm{~S}$ & $37.28 \mathrm{E}$ & 1320 & 5 & 77 & $38.6^{*}$ & 14 \\
\hline Nairobi & $01.17 \mathrm{~S}$ & $36.50 \mathrm{E}$ & 1576 & 3 & 56 & 27.9 & 6 \\
\hline Nakuru & $00.16 \mathrm{~S}$ & $36.04 \mathrm{E}$ & 1860 & 1 & 20 & - & 14 \\
\hline Nanyuki & $00.05 \mathrm{~S}$ & $37.10 \mathrm{E}$ & 2220 & 2 & 40 & $44.0^{*}$ & 12 \\
\hline Ngong Hills & $01.24 \mathrm{~S}$ & $36.38 \mathrm{E}$ & 2460 & 13 & 195 & 20.4 & 5 \\
\hline Shimba Hill & $04.12 \mathrm{~S}$ & $39.28 \mathrm{E}$ & 448 & 1 & 13 & - & 1 \\
\hline Soudu Kisumu & $00.08 \mathrm{~S}$ & $34.47 \mathrm{E}$ & 1151 & 1 & 20 & - & 15 \\
\hline Tunyai & $00.10 \mathrm{~S}$ & $37.50 \mathrm{E}$ & 1029 & 1 & 15 & - & 12 \\
\hline
\end{tabular}


Table I - Continued

\begin{tabular}{|c|c|c|c|c|c|c|c|}
\hline \multirow[b]{2}{*}{$\begin{array}{l}\text { Countries } \\
\text { and localities }\end{array}$} & \multirow{2}{*}{\multicolumn{2}{|c|}{ Co-ordinates }} & \multirow[b]{2}{*}{ Altitude (m) } & \multicolumn{2}{|c|}{ Sample sizes } & \multirow[b]{2}{*}{ Variance } & \multirow[b]{2}{*}{$\begin{array}{l}\text { Map } \\
\text { Ref. }\end{array}$} \\
\hline & & & & Colonies & Bees & & \\
\hline \multicolumn{8}{|l|}{ Lesotho } \\
\hline Ha Lejone & $29.10 \mathrm{~S}$ & $28.27 \mathrm{E}$ & 1479 & 2 & 40 & 24.4 & 7 \\
\hline Mafeteng & $29.48 \mathrm{~S}$ & $27.13 \mathrm{E}$ & 1616 & 7 & 140 & 24.5 & 1 \\
\hline Marakabei & $29.32 \mathrm{~S}$ & $28.08 \mathrm{E}$ & 1977 & 7 & 140 & 35.9 & 6 \\
\hline Mokhotlong & $29.35 \mathrm{~S}$ & $29.17 \mathrm{E}$ & 2133 & 6 & 120 & 30.5 & 2 \\
\hline Quachasnek & $30.06 \mathrm{~S}$ & $20.40 \mathrm{E}$ & 1878 & 7 & 140 & 14.6 & 3 \\
\hline Quiteng & $30.25 \mathrm{~S}$ & $27.42 \mathrm{E}$ & 1578 & 7 & 140 & 12.1 & 4 \\
\hline Sani Top & $29.33 \mathrm{~S}$ & $29.13 \mathrm{E}$ & 2496 & 1 & 20 & - & 9 \\
\hline Semonkong & $29.50 \mathrm{~S}$ & $28.05 \mathrm{E}$ & 2200 & 7 & 140 & 33.0 & 8 \\
\hline Thaba-Tseka & $29.31 \mathrm{~S}$ & $28.35 \mathrm{E}$ & 2286 & 3 & 60 & 23.4 & 5 \\
\hline \multicolumn{8}{|l|}{ Malawi } \\
\hline Blantyre & $15.46 \mathrm{~S}$ & $35.00 \mathrm{E}$ & 1000 & 1 & 15 & - & 4 \\
\hline Chikwawa & $16.02 \mathrm{~S}$ & $34.54 \mathrm{E}$ & 100 & 6 & 120 & 25.4 & 2 \\
\hline Chilinda & $10.36 \mathrm{~S}$ & $33.48 \mathrm{E}$ & 2600 & 26 & 420 & $45.6^{*}$ & 16 \\
\hline Chitipa & $09.41 \mathrm{~S}$ & $33.19 \mathrm{E}$ & 1300 & 6 & 120 & 31.2 & 17 \\
\hline Dedza & $14.20 \mathrm{~S}$ & $34.24 \mathrm{E}$ & 1600 & 6 & 120 & 17.3 & 8 \\
\hline Kasungu & $13.04 \mathrm{~S}$ & $33.29 \mathrm{E}$ & 1070 & 7 & 135 & 16.4 & 11 \\
\hline Lilongwe & $13.58 \mathrm{~S}$ & $33.49 \mathrm{E}$ & 1067 & 1 & 15 & - & 9 \\
\hline Mangochi & $14.30 \mathrm{~S}$ & $35.15 \mathrm{E}$ & 450 & 6 & 120 & 7.0 & 7 \\
\hline Mbalachanda & $11.21 \mathrm{~S}$ & $33.22 \mathrm{E}$ & 1336 & 6 & 120 & 21.3 & 14 \\
\hline Mchinji & $13.48 \mathrm{~S}$ & $32.53 \mathrm{E}$ & 1200 & 6 & 120 & 17.2 & 10 \\
\hline Mzimba & $11.55 \mathrm{~S}$ & $33.39 \mathrm{E}$ & 1330 & 6 & 120 & 10.1 & 13 \\
\hline Nkhotakota & $12.55 \mathrm{~S}$ & $34.19 \mathrm{E}$ & 500 & 6 & 120 & $39.2 *$ & 12 \\
\hline Nsanje & $16.47 \mathrm{~S}$ & $35.15 \mathrm{E}$ & 70 & 6 & 120 & 20.2 & 1 \\
\hline Ntcheu & $14.49 \mathrm{~S}$ & $34.38 \mathrm{E}$ & 1100 & 6 & 120 & 25.6 & 6 \\
\hline Rumphi & $10.59 \mathrm{~S}$ & $33.50 \mathrm{E}$ & 1050 & 6 & 120 & 27.3 & 15 \\
\hline Thyolo & $16.04 \mathrm{~S}$ & $35.09 \mathrm{E}$ & 900 & 6 & 120 & 28.3 & 3 \\
\hline Zomba & $15.22 \mathrm{~S}$ & $35.22 \mathrm{E}$ & 950 & 6 & 120 & 18.7 & 5 \\
\hline \multicolumn{8}{|l|}{ Mozambique } \\
\hline Beira & $19.49 \mathrm{~S}$ & $34.52 \mathrm{E}$ & 0 & 3 & 60 & 6.2 & 5 \\
\hline Inhaminga & $18.24 \mathrm{~S}$ & $35.00 \mathrm{E}$ & 327 & 1 & 11 & - & 7 \\
\hline Manhica & $25.23 \mathrm{~S}$ & $32.49 \mathrm{E}$ & 61 & 1 & 13 & - & 3 \\
\hline Marrocuene & $26.15 \mathrm{~S}$ & $32.40 \mathrm{E}$ & 10 & 1 & 12 & - & 2 \\
\hline Maxixe & $23.51 \mathrm{~S}$ & $35.21 \mathrm{E}$ & 76 & 1 & 18 & - & 4 \\
\hline Mueda & $11.40 \mathrm{~S}$ & 39.31E & 439 & 1 & 20 & - & 9 \\
\hline Pemba & $13.00 \mathrm{~S}$ & $40.29 \mathrm{E}$ & 0 & 1 & 16 & - & 8 \\
\hline Salamanga & $26.29 \mathrm{~S}$ & $32.40 \mathrm{E}$ & 45 & 1 & 12 & - & 1 \\
\hline Sussendenga & $20.35 \mathrm{~S}$ & $33.09 \mathrm{E}$ & 585 & 1 & 8 & - & 6 \\
\hline \multicolumn{8}{|l|}{ Namibia } \\
\hline Ariamsvlei & $28.08 \mathrm{~S}$ & $19.05 \mathrm{E}$ & 774 & 4 & 80 & 31.8 & 12 \\
\hline Karasburg & $28.00 \mathrm{~S}$ & $18.43 \mathrm{E}$ & 1013 & 5 & 89 & 23.6 & 2 \\
\hline Katima Molilo & $17.27 \mathrm{~S}$ & $24.10 \mathrm{E}$ & 946 & 1 & 15 & - & 1 \\
\hline Keetmanshoop & $26.36 \mathrm{~S}$ & $18.08 \mathrm{E}$ & 1773 & 4 & 80 & 14.0 & 3 \\
\hline Maltahöhe & $24.50 \mathrm{~S}$ & $17.00 \mathrm{E}$ & 1340 & 1 & 18 & - & 4 \\
\hline Mariental & $24.36 \mathrm{~S}$ & $17.59 \mathrm{E}$ & 1180 & 4 & 80 & 23.8 & 5 \\
\hline Okahandja & $21.59 \mathrm{~S}$ & $16.58 \mathrm{E}$ & 1439 & 4 & 80 & 17.6 & 9 \\
\hline Otavi & $19.39 \mathrm{~S}$ & $17.20 \mathrm{E}$ & 1414 & 2 & 30 & 6.9 & 10 \\
\hline Otjiwarongo & $20.29 \mathrm{~S}$ & $16.36 \mathrm{E}$ & 1565 & 6 & 110 & $46.6^{*}$ & 8 \\
\hline Seeis & $22.29 \mathrm{~S}$ & $17.39 \mathrm{E}$ & 1610 & 1 & 3 & - & 11 \\
\hline Swakopmund & $22.40 \mathrm{~S}$ & $14.34 \mathrm{E}$ & 0 & 4 & 80 & 25.9 & 7 \\
\hline Windhoek & $22.43 \mathrm{~S}$ & $17.06 \mathrm{E}$ & 1779 & 5 & 100 & 17.3 & 6 \\
\hline \multicolumn{8}{|l|}{ Rwanda } \\
\hline Kigali & $01.56 \mathrm{~S}$ & $30.04 \mathrm{E}$ & 1400 & 4 & 65 & 16.3 & 1 \\
\hline \multicolumn{8}{|l|}{ Somalia } \\
\hline Afgoi & $02.07 \mathrm{~N}$ & $45.02 \mathrm{E}$ & 86 & 1 & 20 & - & 3 \\
\hline Baidoa & $03.04 \mathrm{~N}$ & $43.48 \mathrm{E}$ & 485 & 3 & 60 & 15.4 & 4 \\
\hline Buale & $01.14 \mathrm{~N}$ & $42.36 \mathrm{E}$ & 63 & 4 & 80 & $51.3^{*}$ & 1 \\
\hline Bulo Burti & $03.50 \mathrm{~N}$ & $45.33 \mathrm{E}$ & 158 & 2 & 40 & 23.9 & 5 \\
\hline Dugiuma & $01.20 \mathrm{~N}$ & $42.34 \mathrm{E}$ & 63 & 2 & 40 & 9.8 & 2 \\
\hline \multicolumn{8}{|l|}{ South Africa } \\
\hline Aberdeen & $32.29 \mathrm{~S}$ & $24.03 \mathrm{E}$ & 732 & 3 & 44 & 9.9 & 26 \\
\hline Alexander Bay & $28.40 \mathrm{~S}$ & $16.30 \mathrm{E}$ & 0 & 6 & 120 & 29.9 & 1 \\
\hline Aliwal North & $30.45 \mathrm{~S}$ & $26.45 \mathrm{E}$ & 1317 & 6 & 120 & $40.6^{*}$ & 31 \\
\hline
\end{tabular}


Table I - Continued

\begin{tabular}{|c|c|c|c|c|c|c|c|}
\hline \multirow{2}{*}{$\begin{array}{l}\text { Countries } \\
\text { and localities }\end{array}$} & \multirow{2}{*}{\multicolumn{2}{|c|}{ Co-ordinates }} & \multirow[b]{2}{*}{ Altitude (m) } & \multicolumn{2}{|c|}{ Sample sizes } & \multirow[b]{2}{*}{ Variance } & \multirow{2}{*}{$\begin{array}{l}\text { Map } \\
\text { Ref. }\end{array}$} \\
\hline & & & & Colonies & Bees & & \\
\hline \multicolumn{8}{|l|}{ South Africa (cont.) } \\
\hline Badplaas & $25.58 \mathrm{~S}$ & $30.34 \mathrm{E}$ & 1067 & 6 & 120 & 9.9 & 49 \\
\hline Bitterfontein & $31.03 \mathrm{~S}$ & $18.16 \mathrm{E}$ & 354 & 3 & 60 & 4.8 & 6 \\
\hline Boesmanskop & $32.02 \mathrm{~S}$ & $24.19 \mathrm{E}$ & 1677 & 3 & 60 & 26.7 & 25 \\
\hline Booiskraal & $31.50 \mathrm{~S}$ & $22.36 \mathrm{E}$ & 1360 & 2 & 40 & $45.4^{*}$ & 16 \\
\hline Botterkloof & $31.49 \mathrm{~S}$ & $19.17 \mathrm{E}$ & 720 & 3 & 60 & 35.8 & 10 \\
\hline Britstown & $30.37 \mathrm{~S}$ & $23.30 \mathrm{E}$ & 1122 & 4 & 80 & 28.2 & 20 \\
\hline Burgersdorp & $30.59 \mathrm{~S}$ & $26.20 \mathrm{E}$ & 1481 & 4 & 80 & 15.9 & 33 \\
\hline Calvinia & $31.25 \mathrm{~S}$ & $19.45 \mathrm{E}$ & 980 & 5 & 100 & 29.1 & 9 \\
\hline Clanwilliam & $32.11 \mathrm{~S}$ & $18.54 \mathrm{E}$ & 75 & 6 & 120 & 12.7 & 12 \\
\hline Dordrecht & $31.20 \mathrm{~S}$ & $27.03 \mathrm{E}$ & 1637 & 6 & 120 & 13.2 & 36 \\
\hline Durban & $29.55 \mathrm{~S}$ & $31.00 \mathrm{E}$ & 0 & 5 & 100 & 18.2 & 47 \\
\hline East London & $32.58 \mathrm{~S}$ & $27.55 \mathrm{E}$ & 0 & 6 & 120 & 12.7 & 44 \\
\hline Ficksburg & $28.51 \mathrm{~S}$ & $27.43 \mathrm{E}$ & 1575 & 7 & 139 & 17.9 & 60 \\
\hline Fort Beaufort & $32.48 \mathrm{~S}$ & $26.38 \mathrm{E}$ & 456 & 4 & 80 & $43.8^{*}$ & 61 \\
\hline Garies & $30.30 \mathrm{~S}$ & $18.00 \mathrm{E}$ & 227 & 4 & 79 & 3.7 & 5 \\
\hline Harrismith & $28.18 \mathrm{~S}$ & $29.03 \mathrm{E}$ & 1642 & 6 & 120 & 8.8 & 45 \\
\hline Hoedspruit & $24.21 \mathrm{~S}$ & $30.57 \mathrm{E}$ & 603 & 6 & 120 & 9.8 & 50 \\
\hline Hofmeyr & $31.39 \mathrm{~S}$ & $25.50 \mathrm{E}$ & 1251 & 3 & 60 & 10.0 & 38 \\
\hline Ixopo & $30.08 \mathrm{~S}$ & $30.00 \mathrm{E}$ & 992 & 5 & 100 & 20.1 & 48 \\
\hline Jamestown & $31.07 \mathrm{~S}$ & $26.48 \mathrm{E}$ & 1617 & 3 & 60 & 2.5 & 35 \\
\hline Johannesburg & $26.10 \mathrm{~S}$ & $28.02 \mathrm{E}$ & 1753 & 1 & 20 & - & 51 \\
\hline Klerksdorp & $26.58 \mathrm{~S}$ & $26.39 \mathrm{E}$ & 1301 & 6 & 120 & 20.4 & 52 \\
\hline Ladybrand & $29.12 \mathrm{~S}$ & $27.27 \mathrm{E}$ & 1569 & 7 & 140 & 28.5 & 62 \\
\hline Lutzville & $31.46 \mathrm{~S}$ & $18.21 \mathrm{E}$ & 150 & 5 & 100 & 33.2 & 7 \\
\hline Maclear & $31.04 \mathrm{~S}$ & $28.29 \mathrm{E}$ & 1359 & 7 & 140 & 18.4 & 63 \\
\hline Magaliesberg & $26.00 \mathrm{~S}$ & $27.33 \mathrm{E}$ & 1432 & 5 & 100 & 10.6 & 53 \\
\hline Matatiele & $30.21 \mathrm{~S}$ & $28.51 \mathrm{E}$ & 1466 & 7 & 140 & 14.6 & 64 \\
\hline Mesklip & $29.52 \mathrm{~S}$ & $17.53 \mathrm{E}$ & 759 & 1 & 10 & - & 4 \\
\hline Middelwater & $32.25 \mathrm{~S}$ & $22.04 \mathrm{E}$ & 720 & 2 & 40 & 14.6 & 18 \\
\hline Molteno & $31.22 \mathrm{~S}$ & $26.22 \mathrm{E}$ & 1580 & 4 & 79 & $52.6^{*}$ & 37 \\
\hline Murraysburg & $31.58 \mathrm{~S}$ & $23.47 \mathrm{E}$ & 1158 & 1 & 20 & - & 23 \\
\hline Nababeep & $29.36 \mathrm{~S}$ & $17.46 \mathrm{E}$ & 915 & 4 & 80 & 16.5 & 2 \\
\hline Nelspoort & $32.07 \mathrm{~S}$ & $23.01 \mathrm{E}$ & 1015 & 2 & 40 & 6.9 & 24 \\
\hline Nieuwoudtville & $31.24 \mathrm{~S}$ & $19.06 \mathrm{E}$ & 719 & 5 & 100 & $47.2^{*}$ & 8 \\
\hline Nigel & $26.30 \mathrm{~S}$ & $28.28 \mathrm{E}$ & 1606 & 6 & 120 & 33.1 & 54 \\
\hline Postmasburg & $28.18 \mathrm{~S}$ & $23.05 \mathrm{E}$ & 1311 & 4 & 80 & 28.4 & 19 \\
\hline Pretoria & $25.45 \mathrm{~S}$ & $28.12 \mathrm{E}$ & 1400 & 4 & 60 & 3.3 & 55 \\
\hline Queenstown & $31.52 \mathrm{~S}$ & $27.00 \mathrm{E}$ & 1077 & 11 & 219 & 21.2 & 39 \\
\hline Rhodes & $30.47 \mathrm{~S}$ & $27.57 \mathrm{E}$ & 1700 & 5 & 100 & 18.1 & 65 \\
\hline Richmond & $31.23 \mathrm{~S}$ & $23.56 \mathrm{E}$ & 856 & 2 & 40 & 4.1 & 22 \\
\hline Smithfield & $30.09 \mathrm{~S}$ & $26.30 \mathrm{E}$ & 1400 & 4 & 80 & 21.3 & 30 \\
\hline Sodwana Bay & $27.20 \mathrm{~S}$ & $32.45 \mathrm{E}$ & 0 & 1 & 7 & - & 56 \\
\hline Sonop & $31.57 \mathrm{~S}$ & $19.44 \mathrm{E}$ & 450 & 3 & 60 & 27.2 & 11 \\
\hline Springbok & $29.43 \mathrm{~S}$ & $17.55 \mathrm{E}$ & 1400 & 1 & 10 & - & 3 \\
\hline Springfontein & $30.19 \mathrm{~S}$ & $25.36 \mathrm{E}$ & 1519 & 6 & 120 & 26.6 & 29 \\
\hline Sterkstroom & $31.34 \mathrm{~S}$ & $26.33 \mathrm{E}$ & 1343 & 6 & 120 & $75.5^{*}$ & 40 \\
\hline Steynsburg & $31.20 \mathrm{~S}$ & $25.50 \mathrm{E}$ & 1448 & 3 & 60 & $60.7^{*}$ & 34 \\
\hline Sutherland & $32.24 \mathrm{~S}$ & $20.40 \mathrm{E}$ & 1459 & 6 & 120 & 25.7 & 14 \\
\hline Tarkastad & $32.01 \mathrm{~S}$ & $26.16 \mathrm{E}$ & 1290 & 6 & 120 & 13.8 & 41 \\
\hline Thabazimbi & $24.41 \mathrm{~S}$ & $27.21 \mathrm{E}$ & 1026 & 5 & 100 & 18.0 & 57 \\
\hline Tontelbos & $30.56 \mathrm{~S}$ & $20.23 \mathrm{E}$ & 1122 & 3 & 60 & $49.7 *$ & 15 \\
\hline Underberg & $29.50 \mathrm{~S}$ & $29.22 \mathrm{E}$ & 1550 & 1 & 20 & - & 46 \\
\hline Upington & $28.25 \mathrm{~S}$ & $21.15 \mathrm{E}$ & 836 & 5 & 97 & 13.6 & 13 \\
\hline Venterstad & $30.47 \mathrm{~S}$ & $25.48 \mathrm{E}$ & 1340 & 2 & 40 & 26.3 & 32 \\
\hline Victoria West & $31.25 \mathrm{~S}$ & $23.04 \mathrm{E}$ & 1269 & 3 & 60 & 0.7 & 21 \\
\hline Vonkfontein & $31.56 \mathrm{~S}$ & $21.50 \mathrm{E}$ & 1369 & 2 & 40 & 7.6 & 17 \\
\hline Vryheid & $27.52 \mathrm{~S}$ & $30.38 \mathrm{E}$ & 1189 & 6 & 120 & 19.1 & 59 \\
\hline Warmbaths & $24.53 \mathrm{~S}$ & $28.17 \mathrm{E}$ & 1116 & 6 & 120 & 5.8 & 58 \\
\hline Warrenton & $28.09 \mathrm{~S}$ & $24.47 \mathrm{E}$ & 1198 & 6 & 120 & 18.1 & 28 \\
\hline Wiegenaarspoort & $32.38 \mathrm{~S}$ & $23.12 \mathrm{E}$ & 853 & 2 & 40 & 8.5 & 27 \\
\hline Winburg & $28.37 \mathrm{~S}$ & $27.00 \mathrm{E}$ & 1433 & 6 & 120 & 22.3 & 42 \\
\hline Zastron & $30.18 \mathrm{~S}$ & $27.07 \mathrm{E}$ & 1661 & 6 & 120 & 26.6 & 43 \\
\hline
\end{tabular}

Continued on next page 
Table I - Continued

\begin{tabular}{|c|c|c|c|c|c|c|c|}
\hline \multirow{2}{*}{$\begin{array}{l}\text { Countries } \\
\text { and localities }\end{array}$} & \multirow{2}{*}{\multicolumn{2}{|c|}{ Co-ordinates }} & \multirow[b]{2}{*}{ Altitude (m) } & \multicolumn{2}{|c|}{ Sample sizes } & \multirow[b]{2}{*}{ Variance } & \multirow{2}{*}{$\begin{array}{l}\text { Map } \\
\text { Ref. }\end{array}$} \\
\hline & & & & Colonies & Bees & & \\
\hline \multicolumn{8}{|l|}{ Tanzania } \\
\hline Handeni & $05.25 \mathrm{~S}$ & $38.04 \mathrm{E}$ & 1040 & 5 & 25 & 31.1 & 3 \\
\hline Kahama & $03.48 \mathrm{~S}$ & $32.36 \mathrm{E}$ & 1380 & 5 & 25 & 19.2 & 10 \\
\hline Kiteto & $05.50 \mathrm{~S}$ & $36.50 \mathrm{E}$ & 1750 & 5 & 25 & 22.2 & 2 \\
\hline Kwamtoro-Kondoa & $04.14 \mathrm{~S}$ & $35.23 \mathrm{E}$ & 1200 & 5 & 25 & 27.3 & 6 \\
\hline Lusahanga & $02.55 \mathrm{~S}$ & $31.12 \mathrm{E}$ & 1500 & 5 & 25 & 27.5 & 14 \\
\hline Mt. Kilimanjaro & $03.10 \mathrm{~S}$ & $37.30 \mathrm{E}$ & 2500 & 8 & 140 & $60.6^{*}$ & 12 \\
\hline Magu & $02.31 \mathrm{~S}$ & $33.28 \mathrm{E}$ & 1138 & 5 & 75 & 12.1 & 13 \\
\hline Mlali & $06.19 \mathrm{~S}$ & $36.48 \mathrm{E}$ & 1300 & 1 & 15 & - & 1 \\
\hline Njiro/Arusha/Tengeru & $03.23 \mathrm{~S}$ & $36.40 \mathrm{E}$ & 1390 & 21 & 328 & 6.9 & 11 \\
\hline Nzega & $04.13 \mathrm{~S}$ & $33.11 \mathrm{E}$ & 1200 & 5 & 25 & 18.9 & 9 \\
\hline Pangani & $05.27 \mathrm{~S}$ & $39.00 \mathrm{E}$ & 0 & 5 & 25 & 24.3 & 4 \\
\hline Singida & $04.50 \mathrm{~S}$ & $34.45 \mathrm{E}$ & 1524 & 5 & 25 & 16.7 & 7 \\
\hline Tabora & $05.02 \mathrm{~S}$ & $32.50 \mathrm{E}$ & 1188 & 1 & 15 & - & 8 \\
\hline Tanga & $05.07 \mathrm{~S}$ & $39.05 \mathrm{E}$ & 0 & 3 & 60 & 28.2 & 5 \\
\hline \multicolumn{8}{|l|}{ Uganda } \\
\hline Arapai & $01.45 \mathrm{~N}$ & $33.38 \mathrm{E}$ & 1143 & 1 & 15 & - & 6 \\
\hline Bugoye & $00.17 \mathrm{~N}$ & $30.06 \mathrm{E}$ & 1400 & 1 & 15 & - & 1 \\
\hline Hoima & $01.25 \mathrm{~N}$ & $31.22 \mathrm{E}$ & 1300 & 1 & 15 & - & 2 \\
\hline Kampala & $00.19 \mathrm{~N}$ & $32.35 \mathrm{E}$ & 1198 & 1 & 20 & - & 4 \\
\hline Kitgum & $03.17 \mathrm{~N}$ & $32.54 \mathrm{E}$ & 1000 & 1 & 15 & - & 8 \\
\hline Lira & $02.15 \mathrm{~N}$ & $32.55 \mathrm{E}$ & 1101 & 1 & 15 & - & 7 \\
\hline Masindi & $01.41 \mathrm{~N}$ & $31.45 \mathrm{E}$ & 1147 & 2 & 27 & 9.9 & 3 \\
\hline Mbale & $01.04 \mathrm{~N}$ & $34.12 \mathrm{E}$ & 1300 & 1 & 15 & - & 5 \\
\hline \multicolumn{8}{|l|}{ Zambia } \\
\hline Chipata & $13.40 \mathrm{~S}$ & $32.42 \mathrm{E}$ & 1104 & 2 & 30 & $43.1 *$ & 8 \\
\hline Ikelenge & $11.30 \mathrm{~S}$ & $24.05 \mathrm{E}$ & 1372 & 5 & 100 & 5.7 & 4 \\
\hline Kabompo & $15.25 \mathrm{~S}$ & 24.13E & 1100 & 2 & 30 & 8.6 & 2 \\
\hline Kitwe & $12.48 \mathrm{~S}$ & $28.14 \mathrm{E}$ & 1288 & 7 & 135 & 7.7 & 7 \\
\hline Lusaka & $15.26 \mathrm{~S}$ & $28.20 \mathrm{E}$ & 1296 & 3 & 60 & 18.1 & 1 \\
\hline Mwinilunga & $11.44 \mathrm{~S}$ & $24.24 \mathrm{E}$ & 1250 & 3 & 45 & 7.1 & 5 \\
\hline Nguru/Balovale & $13.30 \mathrm{~S}$ & $23.06 \mathrm{E}$ & 1078 & 1 & 15 & - & 3 \\
\hline Solwezi & $11.28 \mathrm{~S}$ & $26.23 \mathrm{E}$ & 1299 & 5 & 90 & 31.5 & 6 \\
\hline \multicolumn{8}{|l|}{ Zimbabwe } \\
\hline Beit Bridge & $15.00 \mathrm{~S}$ & $30.15 \mathrm{E}$ & 440 & 5 & 100 & 18.9 & 1 \\
\hline Bulawayo & $20.10 \mathrm{~S}$ & $28.43 \mathrm{E}$ & 1390 & 5 & 100 & 35.7 & 2 \\
\hline Glendale & $17.22 \mathrm{~S}$ & $31.05 \mathrm{E}$ & 1150 & 5 & 100 & 20.6 & 7 \\
\hline Harare & $17.43 \mathrm{~S}$ & $24.13 \mathrm{E}$ & 1478 & 14 & 280 & 15.7 & 6 \\
\hline Karoi & $16.46 \mathrm{~S}$ & $29.45 \mathrm{E}$ & 1251 & 6 & 120 & 24.0 & 8 \\
\hline Marondera & $18.11 \mathrm{~S}$ & $31.33 \mathrm{E}$ & 1688 & 4 & 80 & 10.1 & 5 \\
\hline Masvingo & $20.05 \mathrm{~S}$ & $30.50 \mathrm{E}$ & 1099 & 5 & 100 & 10.8 & 3 \\
\hline Mutare & $19.00 \mathrm{~S}$ & $32.40 \mathrm{E}$ & 338 & 6 & 120 & 17.0 & 4 \\
\hline
\end{tabular}

The same nine characters used in previous studies of honeybees in Africa were measured (Crewe et al., 1994; Radloff, 1996; Hepburn and Radloff, 1998). Their Ruttner (1988) numbers are given in parentheses as follows: length of cover hair on tergite $5(1)$, width of wax plate on sternite 3 (11), transverse length of wax plate on sternite 3 (13), pigmentation of scutellum (35), pigmentation of scutellar plate (36), pigmentation of tergite 2 (32), wing angle B4 (22), wing angle N23 (30) and wing angle O26 (31).

Multivariate statistical analysis of the colony mean data included factor analysis and linear discriminant analysis. The latter procedure may provide an overly optimistic estimate of the probability of correct classifi- cation. A jackknife procedure was therefore carried out that classified each colony into a group with the highest a posteriori probability, according to the discrimination functions computed from all the data except the colony being classified (Lachenbruch and Mickey, 1968). Wilk's lambda test was used to compare multivariate population means between groups. The distribution of the statistic was approximated by the F distribution (Mardia et al., 1979). Levene's F-statistic for testing the equality of the variances between groups was also used in the analysis. For the morphometric analyses, colony means, standard deviations and covariances of the morphometric characters were analysed. 
RESULTS

\section{Mesolevel analysis}

In a factor analysis of the morphometric characters of worker honeybees from 825 colonies with a mean sampling distance resolution of $210 \mathrm{~km}$, three factors with eigenvalues greater than one were isolated: factor 1, pigmentation of the scutellum (35) and abdominal tergite 2 (32); factor 2, width and length of wax plate on sternite 3 (11) and (13), factor 3, angles of wing venation N23 (30) and $\mathrm{O} 26$ (31). These factors accounted for $58.8 \%$ of the variance in the data. The factor loadings for each character had absolute values greater than 0.65 . The graph of the factor scores from factors 1 and 2 showed one main morphocluster with colonies from Ethiopia scattered to the left of the main cluster (Figure 2). This result confirms that the honeybees of Ethiopia have darker pigmentation than those from the A. m. scutellata subspecies (Radloff and Hepburn, 1997).
A stepwise discriminant analysis using the colony means of the morphometric characters confirmed the separation of the colonies from Ethiopia from the main cluster. The linear discriminant functions obtained using the most discriminatory characters classified $90.2 \%$ (four misclassified) of the colonies from Ethiopia correctly with a posteriori probabilities $0.69 \leq \mathrm{P} \leq 0.98$ for six colonies and $\mathrm{P}=1.0$ for the remaining colonies and $96.2 \%$ (27 misclassified) of the colonies correctly from the rest of the data set. A jackknife procedure gave the same classification results except that one more colony from the main group was misclassified into the group from Ethiopia. A significant difference was found between the means of the two groups $(\Lambda=0.52$ with $7,1,752$ d.f., $\mathrm{F}=93.97$ with 7,746 d.f., $\mathrm{P}<0.0001)$. The variances of the factor 1 scores and factor 2 scores were used to test for the homogeneity of the colony variance at each locality. A significant difference was found between the intercolonial variances over all the localities (Levene's test, $\mathrm{F}=2.54$ with 184,551 d.f., $\mathrm{P}<0.0001$ ). Those localities with significantly higher variances are indicated in Table I.

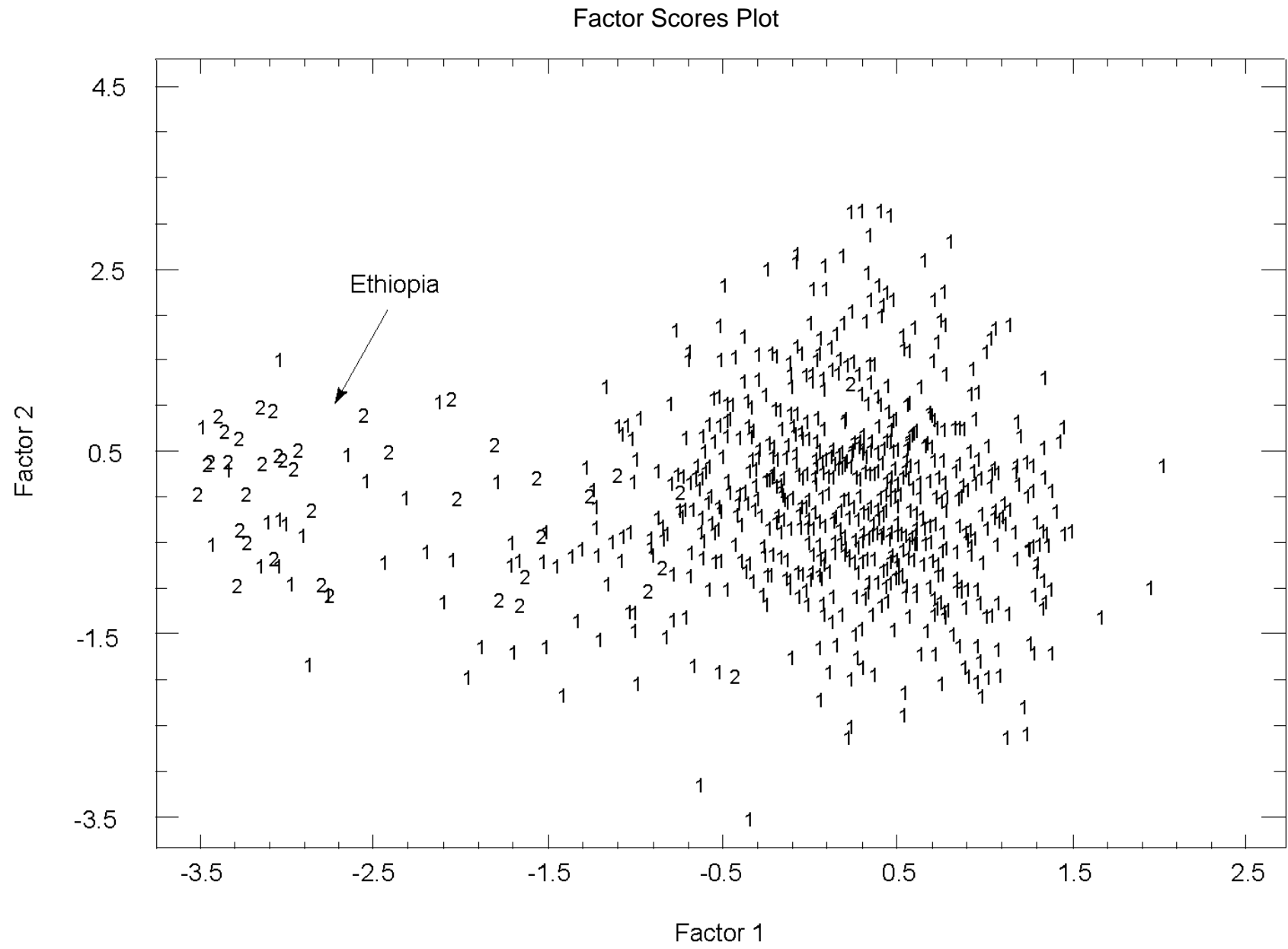

Figure 2 - The graph of the factor scores from factors 1 and 2 shows a large morphocluster to the right of the figure that represents Apis mellifera scutellata bees, and the small cluster to the left represents the honeybees of Ethiopia. 
Distance effects

The length of the transect may obscure small biometric groups if the between-group variation is considerably larger than the within-group variation (Table II). When the colonies from Ethiopia alone were analysed, three morphoclusters were isolated, namely A. m. jemenitica, $A$. $m$. bandasii and $A$. m. scutellata (Radloff and Hepburn, 1997). Also when the colonies from Kenya, Uganda, Tanzania, Rwanda, Burundi, Malawi, Zambia and northern Mozambique were analysed, three morphoclusters were delineated, namely A. m. scutellata, A. m. monticola (black) from the high altitudes of Kenya and Tanzania and $A$. $m$. monticola (yellow) from the high altitudes of the Nyika Plateau, Malawi (Hepburn and Radloff, 1998).

Because the honeybees at higher altitudes in other African mountains (e.g. Mt. Kilimanjaro, Mt. Kenya) differ from lower surrounding populations in both size and pigmentation (Ruttner, 1988), these traits were specifically examined for the honeybees of the Nyika Plateau in Malawi. In this case, there was a significant correlation between size (1) and altitude, that is, bees become increasingly larger with increasing altitude $(\mathrm{r}=0.59, \mathrm{P}<0.0001)$. There was also a significant correlation between pigmentation (35) and altitude; with increasing altitude the bees became lighter in colour $(\mathrm{r}=0.44, \mathrm{P}<0.0001)$.

Finally, statistical comparisons of high-altitude bees from the Nyika Plateau with others from Mt. Kilimanjaro (Tanzania), Mt. Kenya (Kenya) and the Drakensberg mountains revealed all of these bees to be larger in size than their lower-altitude counterparts ( $F=10.75$ with 4,297 d.f., $\mathrm{P}<0.0001$ ); however, the honeybees of the Drakensberg and Nyika Plateau are significantly lighter in colour than the more northerly mountain bees $(F=109.00$ with 3,82 d.f., $P<0.0001$ ).

\section{Variance characteristics}

The intercolonial variance values of the populations sampled are listed in Table I. Only 19 of the 193 localities (about 10\%) exhibited statistically significant elevated values of variance. It is of interest to consider the sources of the high variance domains. The intercolonial variance is derived from mean values of whole colonies (between colonies) for each locality (Table I) but it is not immediately obvious what different components give rise to the variance. A plot of those high variance colonies on an intracolonial (within colonies) basis shows that high variance values arise in two different ways. Of 19 high intercolonially variant colonies, 10/19 exhibited a range of variances that statistically yield high variance, but there was no single colony in the locality set which exhibited significantly high within-colony variance (Table III). Thus, for these colonies the localised population was highly heterogeneous. The remaining $9 / 19$ colonies yielded high intercolonial variances because particular individual colonies were themselves highly variant. In fact all nine of this second group of localities were correlated with hybrid zones based on morphocluster analysis and were indicative of hybridisation. Thus there are two separate origins for high

Table II - The effects of sampling distance intervals on morphocluster inclusiveness.

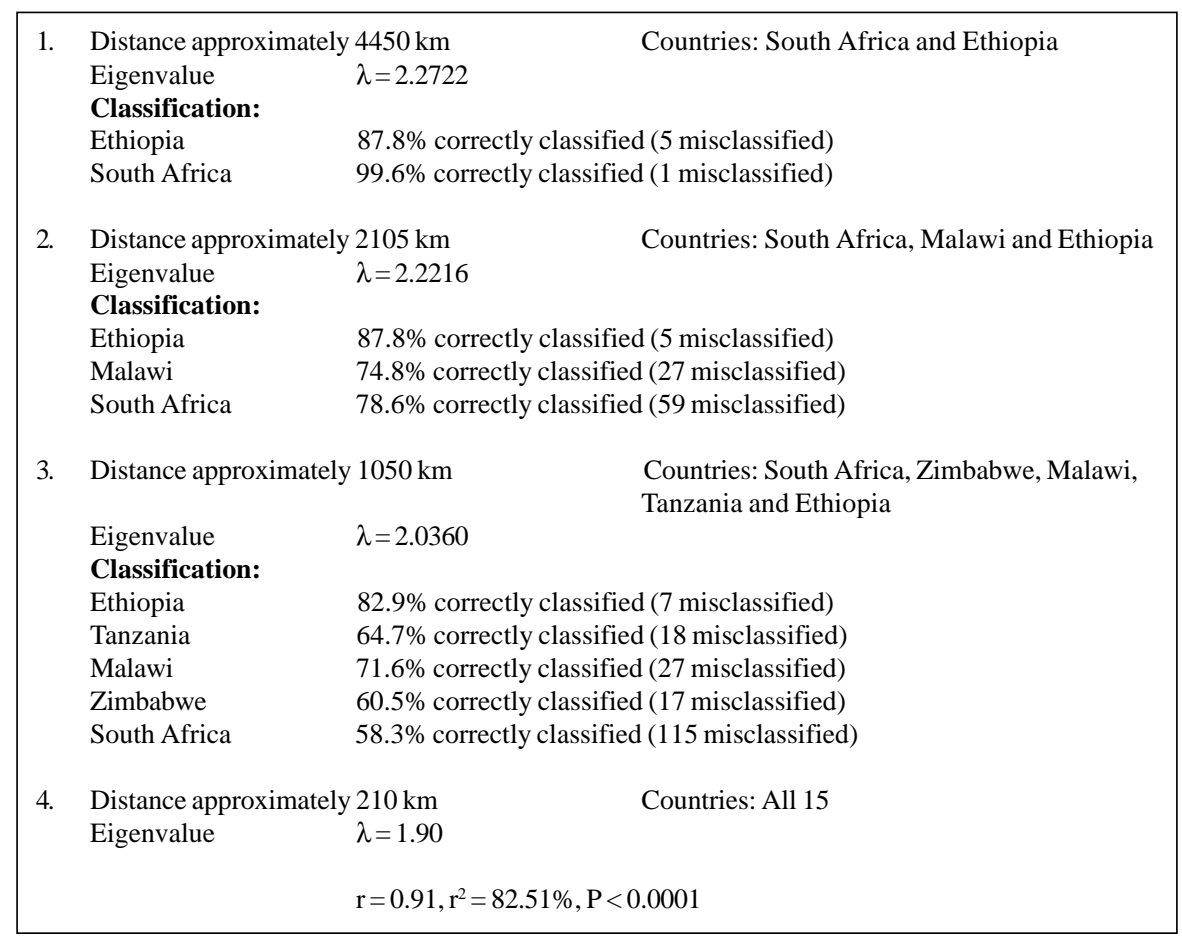


Table III - Intracolonial variances of worker honeybees at localities with high intercolonial variances.

\begin{tabular}{|c|c|c|}
\hline $\begin{array}{l}\text { Countries and } \\
\text { localities }\end{array}$ & Individual colony variances & $\begin{array}{c}\text { Combined colony } \\
\text { variance }\end{array}$ \\
\hline \multicolumn{3}{|l|}{ Burundi } \\
\hline Bujumbura & $38.72 / 29.98 / 31.98 / 41.76 / 56.74 * / 54.67 *$ & 72.3 \\
\hline \multicolumn{3}{|l|}{ Kenya } \\
\hline Chiokariga & $32.16 / 26.89 / 31.13$ & 46.1 \\
\hline Meru & $33.90 / 27.43 / 49.52 * / 32.24$ & 42.2 \\
\hline Mt. Kenya & $27.02 / 34.97 / 26.05 / 25.13 / 22.83$ & 42.4 \\
\hline Nanyuki & $20.14 / 19.19$ & 37.1 \\
\hline \multicolumn{3}{|l|}{ Malawi } \\
\hline \multirow[t]{4}{*}{ Chilinda } & $34.70 / 27.29 / 19.49 / 25.67 / 33.25 / 33.58 / 29.83$ & 41.9 \\
\hline & $43.52 / 37.77 / 39.83 / 27.47 / 36.78 / 31.64 / 19.97$ & \\
\hline & $23.19 / 35.47 / 39.39 / 30.05 / 23.53 / 34.11 / 29.62$ & \\
\hline & $31.22 / 34.08 / 18.12 / 41.97 / 26.48$ & \\
\hline Nkhotakota & $31.43 / 35.47 / 38.87 / 27.38 / 33.40 / 23.16$ & 44.9 \\
\hline \multicolumn{3}{|l|}{ Namibia } \\
\hline Otjiwarongo & $31.34 / 28.78 / 25.47 / 29.77 / 43.61 / 37.47$ & 53.0 \\
\hline \multicolumn{3}{|l|}{ Somalia } \\
\hline Buale & $23.09 / 35.29 / 29.69 / 27.47$ & 53.1 \\
\hline \multicolumn{3}{|l|}{ South Africa } \\
\hline Aliwal North & $39.32 / 25.65 / 60.98 * / 62.19 * / 67.55 * / 37.49$ & 62.7 \\
\hline Booiskraal & $33.34 / 67.00^{*}$ & 61.5 \\
\hline Fort Beaufort & $75.86^{*} / 51.88^{*} / 43.62 / 63.58 *$ & 69.9 \\
\hline Molteno & $48.46 / 73.34 * / 40.37 / 59.91 *$ & 72.4 \\
\hline Nieuwoudtville & $27.40 / 53.25 * / 31.92 / 33.55 / 28.74$ & 55.4 \\
\hline Sterkstroom & $56.69 * / 35.37 / 41.99 / 42.56 / 49.44 * / 341.57 *$ & 158.4 \\
\hline Steynsburg & $38.84 / 40.24 / 44.57$ & 64.4 \\
\hline Tontelbos & $34.40 / 65.94 * / 89.88 *$ & 76.7 \\
\hline \multicolumn{3}{|l|}{ Tanzania } \\
\hline Mt. Kilimanjaro & $26.54 / 35.68 / 37.06 / 24.17 / 48.48 / 37.55 / 30.59 / 47.82$ & 60.4 \\
\hline \multicolumn{3}{|l|}{ Zambia } \\
\hline Chipata & $32.45 / 34.92$ & 45.4 \\
\hline
\end{tabular}

* Significantly higher variance $(\mathrm{P}<0.05)$.

intercolonial variance: those colonies in which the bees are themselves heterogeneous (intracolonial variance) and other colonies where the within-colony variance is not significantly high but collectively the colonies of bees of the locality are significantly more variant than others from neighbouring localities. Thus, of the 193 colonies of honeybees analysed in this way nearly $90 \%$ of them are fairly homogeneous as morphocluster entities.

The geographical distributions of these high intercolonial variances demonstrate distinct patterns. For example, all of the localities marked with an asterisk in Table I in Burundi, Kenya, Tanzania and Malawi are associated with mountain systems for which the "A. m. monticolalike" bees occur in an archipelago surrounded by the morphometrically more uniform and distinct $A$. $m$. scutellata. In the case of South Africa, similarly marked variances (except two unexplained cases) all came from the natural hybrid zone between A. m. capensis and A. m. scutellata in mountainous countryside. Those colonies of Zambia and Namibia with high variances are in hybrid zones between A. m. scutellata and A. m. adansonii. Too little information is available on the bees of Somalia (Buale) to even venture comment at this stage.

Those colonies exhibiting statistically high levels of intercolonial variance between localities could also be related to geophysical parameters. Figure 3 depicts regions of high variance against altitudinal relief in the $A . \mathrm{m}$. scutellata area. It is evident that the majority of high variance localities are associated with areas of greatest rate of altitudinal change throughout eastern Africa. Altitude of course reflects modification of climatic systems and consequential to this are changes in vegetation structure of differing biomes and to the bees themselves (Hepburn et al., 1998).

Figure 4 depicts the high variance localities of $A$. m. scutellata in eastern Africa on a map of the major climatic zones of the continent. Here it becomes strikingly evident that high variance is typically associated with regions of climatic transition in most instances. Those high variance localities not in such transitional zones are nonetheless associated with more localised discontinuities in climate for which there are significant differences in heat and/or cold intensity regimes. Figure 5 depicts the high variance localities against the major vegetation zones of eastern Africa and again it can be seen that the local populations of these bees are associated with edge effects, which are transitional regions between the major biomes. In a final biogeographical composite the regions of 

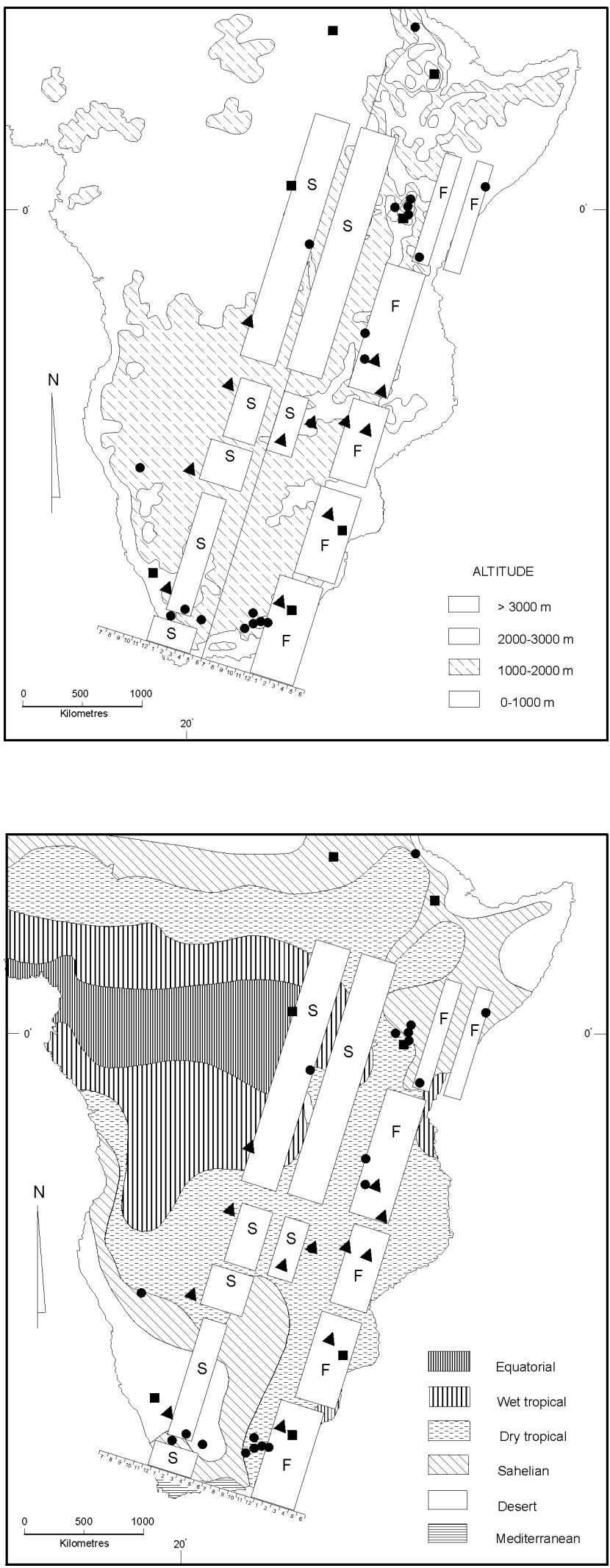

Figure 4 - Map of the regions of high morphometric variance in relation to the major climatic zones of east Africa. Symbols as in Figure 3. (Map modified from van Chi-Bonnardel, 1973).
Figure 3 - Relief map of eastern Africa indicating areas (circles) of significantly high values of morphometric variance within the Apis mellifera scutellata populations. (Map modified from van Chi-Bonnardel, 1973). Triangles denote localised regions of high temperature changes, squares denote low temperature discontinuities. Principal reproductive swarming ( $S$ ) and major flowering $(\mathrm{F})$ periods of the relevant bee flora are indicated to the left and right of the vertical line, respectively. Horizontal scale units are months of the year beginning on both sides of the vertical line with July (month 7) and running through June (month 6) because July is mid-winter and the end of the annual colony cycles. The map is diagrammatic for swarming and flowering as they change with latitude.

high variance for the A. m. scutellata populations are illustrated in terms of localised discontinuities of climate (rapid changes in hot or cold in a small region) and also in relation to the principal swarming seasons and the principal flowering periods for the relevant major honeybee flora of eastern Africa. The scale at the bottom of the map represents months of the year for swarming (left side of vertical line) and for flowering (right side of vertical line) and both are expressed as months of the year beginning with July and ending with June (months 7 to 6 ) because July is the winter end of the annual cycle. The drawing is diagrammatic for an imaginary vertical line running north-south through the region so that swarming and flowering are averaged for the eastern part of the continent. Each must be read as running horizontally across the vertical line for any particular latitude in eastern Africa. Seen in this way the honeybee populations of southern Africa (8 high variance regions) enjoy more or less equality of flowering periods but a narrower window of reproductive swarming. However, note that swarming and seasonal flowering are geographically related to localised climatic discontinuities which result in ecological instability at the edges. This should in turn lead to selective pressure for a high turnover in gene flow and partially explain the high levels of variance associated with such regions.

\section{DISCUSSION}

Two principal conclusions about the morphocluster analysis emerge in this study. Firstly the graph of the fac- 


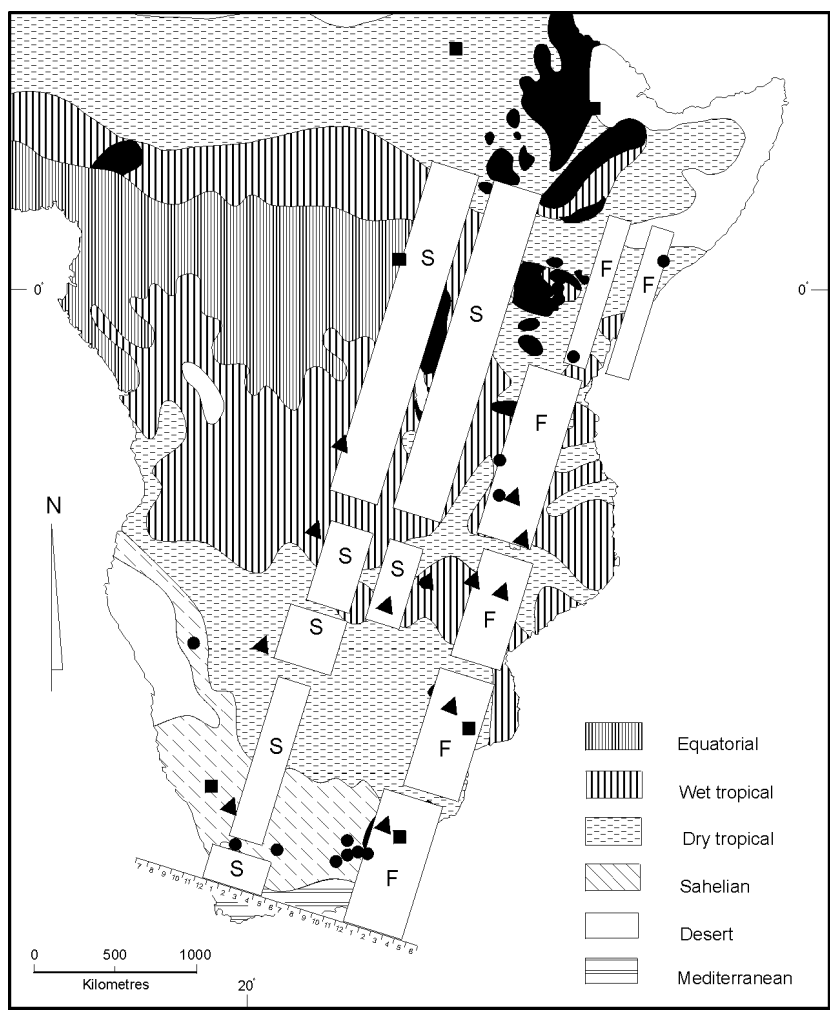

Figure 5 - Map of regions of high morphometric variance in relation to the major zones of vegetation (biomes) in eastern Africa. High variance domains are primarily associated with transitional areas between biomes. Symbols as in Figure 3. (Map modified from van Chi-Bonnardel, 1973).

tor scores (Figure 2) confirm that the honey bees of Ethiopia are morphometrically distinguishable from all of the other more southerly bees, all of which have been previously defined as A. m. scutellata. Secondly, detailed and localised analyses of the mountain populations of the honeybees of eastern Africa show that they can indeed be differentiated, to a greater or lesser extent, from the A. $m$. scutellata that surround the mountain archipelago bees. However, it is now evident that the mountain populations themselves can in fact be further differentiated into different groups. Because of the lack of precision in the traditional usage of terms such as "subspecies" or "ecotype" we simply note what morphoclusters can be formed and do not assign names to them.

The results of Table II show that the greater the distance between countries, the greater the extent of variation in morphometric characters. As a corollary, the greater the distance between countries, the higher the probability of "correctly" assigning colonies to specific morphoclusters. This conforms exactly with conclusions reached in studies on the effects of sampling distance and variable confidence limits (Radloff and Hepburn, 1998): the greater the distance between samples, the more distinct the morphoclusters.

The significance of the sampling distance interval is clearly demonstrated in analyses of localised regions. For example, the discriminant analysis of the morphometric characters of the honeybees of eastern Africa unequivocally established the occurrence of two distinct morphoclusters. One morphocluster comprises honeybees living at high altitudes $( \pm 2500 \mathrm{~m})$ on the Nyika Plateau (Malawi) while a second morphocluster comprised all of the bees at altitudes below $\pm 1600 \mathrm{~m}$ throughout Malawi from the borders of Tanzania to Mozambique. Following the system of classification of Ruttner (1988) the lower altitude bees are A. $m$. scutellata Lepeletier and the high ones A. $m$. monticola Smith.

The mountain bees of Nyika bear close morphological similarities to those of other mountains such as Mt. Kenya, Mt. Meru and Mt. Elgon to the north and to others of the more southerly Drakensberg (Hepburn and Radloff, 1998; Radloff and Hepburn, 1998). The Nyika bees are significantly larger in size than those of lower altitude. However, pigmentation presents some interesting problems. With increasing altitude the southern mountain bees (Nyika and Drakensberg) become more yellow in overall colouration while in the northern mountain bees the trend is to darker colouration with increasing altitude. It is possible that the high mountain bees do in fact constitute a unique subspecies $A$. m. monticola distinct from $A$. $m$. scutellata at lower altitudes as proposed by Ruttner (1988) and Meixner et al. (1989). However, final resolution of this problem will require a critical DNA analysis.

Morphometric variance among colonies of a natural honeybee population can be attributed to two proximate causes. Because queen honeybees are polyandrous (Adams et al., 1977; Neumann et al., 1999, 2000), honeybee colonies may consist of several to many patrilines. The effect is that regions of high variance of either or both high intracolonial variance among workers as well as intercolonial variance may occur. However, variance must be seen in the broader context of frequency distributions of character states.

The genetics of metric character states such as can be derived from morphometrics of honeybee centres around the analysis of the frequency distribution patterns of variation for it is in terms of variation that primary population genetic questions can be formulated (Wright, 1969, 1978; Falconer and Mackay, 1997). The basic premise underlying the analysis of variation is that it can be partitioned into components of differing probable cause. The relative magnitude of these components determine the genetic properties or structure of populations and the extent of this variation is expressed in terms of variance.

A final comment on the A. $m$. scutellata that were introduced into the Neotropics can now be made. Kerr (1992) clearly stated where all of this original honeybee livestock originated, principally the Transvaal region of South Africa. It can be noted in the present set of results that the A. $m$. scutellata bees of that area are extremely homogeneous and display low variance values, and are uniformly aggressive and virulently invasive. Thus, it can be concluded 
from the analysis of the mother African material alone that those A. $\mathrm{m}$. scutellata that spread through the Neotropics did so on the basis of a founder effect. This conclusion is absolutely compatible with the identical conclusion reached on mitochondrial studies of $A$. m. scutellata in the Neotropics (Smith et al., 1999; Del Lama, 1999).

\section{ACKNOWLEDGMENTS}

We thank Dr P. Neumann for reviewing the manuscript.

\section{RESUMO}

Populações africanas de abelhas comuns classificadas como Apis mellifera scutellata Lepeletier foram analisadas morfometricamente usando-se técnicas estatísticas multivariadas. A população consistia de aproximadamente 15.000 abelhas operárias provenientes de 825 colônias individuais de 193 localidades do leste da África, estendendo-se da África do Sul até a Etiópia. A análise de fatores estabeleceu um agrupamento primário designado $A$. $m$. scutellata. A formação de agrupamento morfológico e a inclusividade (classificação correta) são altamente sensíveis aos intervalos de distância da amostragem. Dentro da região de A. m. scutellata há abelhas maiores associadas às altas altitudes montanhosas, que são tradicionalmente classificadas como A. m. monticola Smith, masé evidente que estas abelhas não formam um grupo uniforme. As características de variação das medidas morfométricas mostram domínios de populações locais significantemente diferentes. Estas populações altamente variáveis ocorrem em sua maioria em margens de transição de zonas climáticas e de vegetação, e algumas vezes com alterações mais localizadas de temperatura. Agora também é evidente que as A. $m$. scutellata introduzidas há aproximadamente 50 anos na região neotropical constituíam uma amostra particularmente homogênea que exibia todos os caracteres esperados em uma população com efeito fundador ou "gargalo".

\section{REFERENCES}

Adams, J., Rothmann, E.D., Kerr, W.E. and Paulino, Z.L. (1977). Estimation of the number of sex alleles and queen matings from diploid male frequencies in a population of Apis mellifera. Genetics 86: 583-596.

Crewe, R.M., Hepburn, H.R. and Moritz, R.F.A. (1994). Morphometric analysis of 2 southern African races of honeybees. Apidologie 25: 61-70.
Daly, H.V. (1991). Systematics and identification of Africanized honey bees. In: The "African" Honey Bee (Spivak, M., Fletcher, D.J.C. and Breed, M.D., eds.). Westview Press, Boulder, pp. 13-44.

Del Lama, M.A. (1999). Population genetics of the Africanized honeybees from South America. In: Proceedings of the XIII International Congress of IUSSI, Adelaide, p. 133.

Falconer, D.S. and Mackay, T.F.C. (1997). Introduction to Quantitative Genetics. Longman, Essex.

Hepburn, H.R. and Radloff, S.E. (1998). Honeybees of Africa. Spinger Verlag, Berlin.

Hepburn, H.R., Radloff, S.E., Brown, R.E., Youthed, C. and Illgner,P. (1998) Production of aerodynamic power in mountain honeybees (Apis mellifera). Naturwissenschaften 85: 389-390.

Kerr, W.E. (1992). Abejas Africanas su introduccion y expansion en el continente Americano. Subespecies y ecotipos Africanos. Ind. Apic. 13: $12-21$

Kerr, W.E. and Portugal-Araújo, V. De (1958). Raças de abelhas da Africa. Garcia de Orta 6: 53-59.

Lachenbruch, P. and Mickey, R.M. (1968). Estimation of error rates in discriminant analysis. Technometrics 10: 1-11.

Mardia, K.V., Kent, J.T. and Bibby, J.M. (1979). Multivariate Analysis. Academic Press, London.

Meixner, M., Ruttner, F., Koeniger, N. and Koeniger, G. (1989). The mountain bees of the Kilimanjaro region and their relation to neighbouring bee populations. Apidologie 20: 165-174

Neumann, P., Moritz, R.F.A. and Van Praagh, J. (1999). Queen matingfrequency in different types of honeybee mating apiaries. J. Apic. Res. 38: 11-18.

Neumann, P., Moritz, R.F.A., Radloff, S.E. and Kryger,P. (2000). Heritability for polyandry in the honeybee (Apis mellifera L.). Heredity (in press).

Radloff, S.E. (1996). Multivariate analysis of selected honeybee populations in Africa. $\mathrm{PhD}$ thesis, Rhodes University, Grahamstown.

Radloff, S.E. and Hepburn, H.R. (1997). Multivariate analysis of honeybees, Apis mellifera Linnaeus (Hymenoptera: Apidae) of the Horn of Africa. Afr. Entomol. 5: 57-64.

Radloff, S.E. and Hepburn, H.R. (1998). The matter of sampling distance and confidence levels in the subspecific classification of honeybees, Apis mellifera L. Apidologie 29: 491-501.

Ruttner, F. (1988). Biogeography and Taxonomy of Honeybees. Springer Verlag, Berlin.

Smith, F.G. (1961). The races of honeybees in Africa. Bee World 42: 255-260.

Smith, D.R., Palmer, M.S., Orive, M.E. and Taylor, O.R. (1999). Modeling founder effects in Neotropical African honey bees. In: Proceedings of the XIII International Congress of IUSSI, Adelaide, p. 449.

van Chi-Bonnardel, R. (1973). The Atlas of Africa. Jeune Afrique, Paris.

Wright, S. (1969). Evolution and Genetics of Populations. Vol. 2. The Theory of Gene Frequencies. University of Chicago Press, Illinois.

Wright, S. (1978). Evolution and Genetics of Populations. Vol. 4. Variability Within and Among Natural Populations. University of Chicago Press, Illinois.

(Received March 18, 1999) 